\title{
Early antioxidant supplementation in the prevention of diabetic nephropathy: alterations in NOS expression precede renal functional decline
}

\begin{abstract}
Rat and human studies have shown that good glycemic control alone is not sufficient to prevent the complications associated with diabetes, such as nephropathy. A proposed culprit is excessive oxidative stress in the cells and vasculature of the kidney resulting in endothelial cell dysfunction, which is one of the earliest and most pivotal consequences seen in early diabetes. We hypothesized that an antioxidant (AO) diet would alleviate the oxidative stress and prevent and/or delay the progression of diabetic nephropathy, even with poor glycemic control. Early nephropathy in diabetes is associated with increased intra renal nitric oxide (NO) production. Nitric oxide synthases (NOS) are a family of enzymes that catalyze the production of NO. We report significantly lower endothelial nitric oxide synthase (eNOS) with $\mathrm{AO}$ supplementation compared to the other 2 groups, which means the AO diet led to the decrease in eNOS in the early phase of the onset of diabetic nephropathy. Also, we noted a significant decrease in neuronal nitric oxide synthase (nNOS) with $\mathrm{AO}$ supplementation, which is consistent with renal protection in the later phase of diabetic nephropathy. Our results reveal evidence of molecular damage to the endothelium and specialized structures of the kidney that precedes the onset of diabetes. These results suggest early AO supplementation safeguards the kidneys from some of the forces that induce hyperfiltration in early-stage diabetic nephropathy.
\end{abstract}

Keywords: diabetes, nephropathy, renal function, kidney, glycemic control, antioxidants
Volume 4 Issue I - 2017

\author{
Lubka B Ilieva, 1,2 Taylor Claybaugh, 1,2 Jean R \\ Thuma,' Yuriy Slyvka,' Sharon Inman,' Kelly \\ D McCall ${ }^{1-5}$ \\ 'Diabetes Institute, Ohio University, Heritage College of \\ Osteopathic Medicine, USA \\ ${ }^{2}$ Center for Osteopathic Research and Education, Ohio \\ University College of Arts \& Sciences, USA \\ ${ }^{3}$ Department of Specialty Medicine, Ohio University, Heritage \\ College of Osteopathic Medicine, USA \\ ${ }^{4}$ The Diabetes Institute at Ohio University, USA \\ ${ }^{5}$ Department of Internal Medicine, Yale University School of \\ Medicine, USA
}

Correspondence: Kelly D McCall, Department of Specialty Medicine, Ohio University, 302B Academic \& Research Center, Athens, OH 4570I, USA, Tel (740) 5930926, Fax (740) 597I37I, Email mccallk@ohio.edu

Received: June 29, 2017 | Published: July II, 2017
Abbreviations: AO, antioxidant; NO, nitric oxide; NOS, nitric oxide synthases; eNOS, endothelial nitric oxide synthase; Nnos, neuronal nitric oxide synthase; T2DM, type 2 diabetes mellitus; ESRD, end-stage renal disease; FBG, fasting blood glucose; HS, high sucrose; GFR, glomerular filtration rate; $\mathrm{CC}$, creatinine clearance

\section{Introduction}

Diabetes imposes an enormous public and economic burden on our population and nation. In adults, type 2 diabetes mellitus (T2DM) accounts for approximately $90 \%$ of all diagnosed cases of diabetes. ${ }^{1}$ Diabetic nephropathy is the leading cause of end-stage renal disease (ESRD) in the United States, accounting for more than 50\% of new cases of renal failure. ${ }^{2}$ It was previously believed that good glycemic control could prevent the complications associated with diabetes: retinopathy, neuropathy, and nephropathy. However, it has been shown that some animal models and patients with T2DM still eventually develop diabetic complications, including nephropathy, despite good glycemic control. ${ }^{3}$

One of the proposed mechanisms thought to cause these complications is oxidative stress. Excessive oxidative stress in the cells and vasculature of the kidney results in endothelial cell dysfunction, which is one of the earliest and most pivotal metabolic consequences leading to vasodilation seen in early diabetes. ${ }^{4}$ Nitric oxide synthases (NOS) are a family of enzymes that catalyze the production of nitric oxide (NO) from L-arginine. In mammals, two constitutive isoforms of NO synthase (NOS) are expressed in the kidney. The endothelial isoform (eNOS) is the primary signal generator in the control of vascular tone in the endothelium of the afferent and efferent arterioles and the glomerular capillaries. The neuronal isoform (nNOS) is expressed in great proportion in some of the specialized structures of the kidney, such as the macula densa cells. When induced under conditions of stress, such as oxidative damage, NOS enzymes undergo extensive post-translational modification that yields a number of different dimers and monomers. ${ }^{5}$

The net effect on renal NO production depends on the mechanisms that prevail in a given stage of the disease. Based on the current evidence, early nephropathy in diabetes is associated with increased intra renal NO production mediated primarily by constitutively released eNOS and nNOS. The enhanced NO production may contribute to hyper filtration and micro albuminuria that characterizes early diabetic nephropathy. ${ }^{6,7}$ On the other hand, a majority of the studies indicate that advanced nephropathy leading to severe proteinuria, declining renal function, and hypertension is associated with a state of progressive NO deficiency. In summary, early oxidative stress causes increased production and secretion of NOS, and its multimers tend to break down into active monomers. After prolonged periods of stress, however, the monomers of NOS are depleted. ${ }^{8}$

Antioxidants (AOs) can prevent pathological changes in kidney disease models, including animal and human diabetic models. Animal studies have shown that AOs prevent glucose-induced activation of many factors that are instrumental in endothelial cell injury. ${ }^{9}$ However, oxidative imbalance may start well before there is evidence of overt nephropathy, and studies have shown a benefit of AOs on preventing or delaying the development of diabetic microvascular 
complications. ${ }^{10,11}$ For example, when AOs are started on the day of streptozotocin induction of T1DM in rats, renal function is preserved. ${ }^{12}$ And early treatment with vitamins $\mathrm{C}$ and $\mathrm{E}$ plus insulin has shown to significantly lower fasting blood glucose (FBG) in this rat model. ${ }^{13}$

Therefore, we hypothesize that despite good glycemic control, kidney damage will still occur and alleviating the oxidative stress in a type 2 diabetic model using an antioxidant diet will prevent and/or delay the progression of diabetic nephropathy even with poor glycemic control. To test this hypothesis, we utilized the Wistar rat model of T2DM, and evaluated the effects of dietary AO supplementation on fasting blood glucose, body weight, markers of oxidative stress and renal function. We report that an antioxidant diet appears to induce a protective decrease in the level of $\mathrm{NO}$ and alleviates the oxidative stress in T2DM.

\section{Materials and methods}

\section{Type 2 diabetic rat model and groups}

All animal work was conducted with approval from the Ohio University Institutional Animal Care and Use Committee.

24 Male Wistar rats were purchased from Harlan Laboratories (Indianapolis, IN) at 4-5weeks of age. After being acclimated to laboratory conditions for 48 hours, they were randomly divided into three groups ( $\mathrm{n}=8 \mathrm{rats} /$ group). Group 1 served as the non-diabetic control and was fed a standard ad libitum diet (Purina Mills, Inc., St. Louis, MO, \#5012). Group 2 served as the type 2 diabetic control and was fed a $65 \%$ high-sucrose (HS) diet known to induce T2DM (Purina Mills, Inc., St. Louis, MO, \#57FB). Group 3 served as the experimental group and was fed the same HS diet mixed 1:1 with an antioxidant diet (Purina Mills, Inc., St. Louis, MO, \#5TVN, Table 1). Each group was maintained on their respective diets and had access to water ad libitum for a total of 12 weeks.

Table I Primary contents of the AO diet; Outline of contents in the antioxidant diet

\begin{tabular}{ll}
\hline Antioxidant(AO) & AO diet \\
\hline Alpha-tocopherol (IU/kg) & 100 \\
Beta-carotene(ppm) & 10.8 \\
Selenium(ppm) & 0.69 \\
Zinc(ppm) & 150 \\
Manganese(ppm) & 150 \\
Copper(ppm) & 12 \\
\hline
\end{tabular}

\section{Testing protocol}

At weeks 0,6, and 12, the animals were placed in metabolic cages to collect 12 hour urine specimens and FBG measurements using a One Touch Glucometer (Johnson and Johnson). At these intervals, their weights were also measured. During the 12 hours of collection, the rats had access to water only ad libitum. Measurements from weeks 0,6 , and 12 were compared with corresponding measurements of renal function. We evaluated renal function using multiple markers. Glomerular filtration rate (GFR), based on creatinine clearance, was determined using urine and plasma creatinine assay kits (Cayman Chemical, Ann Arbor, MI \#500701 and \#700460), and urine output levels. GFR per $100 \mathrm{~g}$ of body weight was calculated by: urine concentration multiplied by urine output and $100 \mathrm{~g}$, all divided by plasma concentration multiplied by the weight of the rat. ${ }^{14}$ Protein concentration was determined using a rat urinary protein assay kit (Chondrex, Inc., Redmond, WA \#9040). These measurements were collected at weeks 0,6 , and 12 weeks.

\section{Post-euthanasia analyses}

At the end of the 12 week period, the rats were euthanized and one kidney was removed, the renal medulla and renal cortex were separated, collected, and stored in liquid nitrogen for renal eNOS and nNOS protein level analyses by Western blot.

\section{Western blot analyses of eNOS and nNOS}

Western blots of the collected kidney samples were performed to detect the levels of eNOS and nNOS isoforms. Kidney cortex and medulla were homogenized in HEPES/sucrose buffer containing proteinase inhibitors and centrifuged at $8000 \mathrm{~g}$ for $20 \mathrm{~min}$ at $4^{\circ} \mathrm{C}$. Electrophoresis was performed in unboiled conditions at $4^{\circ} \mathrm{C}$ on $5-15 \%$ Tris-HCl SDS-PAGE gradient gels. Total protein concentration was detected using a Bicinchoninic Protein Assay Kit (Pierce, Rockford, IL) and equal amounts of protein ( $30 \mu \mathrm{g} / \mathrm{lane})$ were loaded. The proteins in the gels were then transferred overnight onto a PVDF membrane. After transfer, each membrane was cut into two sections. The upper part was developed with one of the NOS-specific antibodies and the lower part with $\beta$-actin-specific-antibody that was used as internal control. We applied polyclonal antibodies to $\operatorname{nNOS}(1: 10,000$ dilution, \#7155, Sigma-Aldrich Corporation, St. Louis, MO) and eNOS (1:1000 dilution, SC-654, Santa Cruz Biotech, Santa Cruz, CA) with no cross reactivity among the different isoforms. The goat antirabbit horseradish peroxidase conjugated secondary antibody (1:2000 dilution, \#20320, Alpha Diagnostics International, San Antonio, TX) was applied. The $\beta$-actin was measured as an internal control using a monoclonal primary antibody (1:2000 dilution, A2228, SigmaAldrich Corporation, St. Louis, MO) and horseradish peroxidaseconjugated goat anti-mouse secondary antibody (1:30,000 dilution, A9044, Sigma-Aldrich Corporation, St. Louis, MO). Membranes were developed with ECL immunoblotting detection reagents with quantification on a Chemi Doc chemi luminescent detection system and Quantity One software (Bio-Rad, Richmond, CA). The results were expressed as NOS $/ \beta$-actin density ratio.

\section{Statistical analysis}

As the main analytic framework multilevel modeling was applied to all dependent variables. Each model contained one betweensubjects factor treatment (TX: Control, Diabetic Control, AO diet) and one within subjects factor time. In each model the baseline level was included as a covariate to increase power to detect group differences. At each time point pair wise comparisons between all possible pairs of groups were performed. The significance level was set to 0.05 . In the figures data are presented as means \pm standard errors.

\section{Results}

\section{Mean body weight}

The three groups of rats gained weight at different rates throughout the experimental timeline. Weeks 6 and 12 show proportional increases in mean body weight between the 3 groups Figure 1. Significant differences between the groups after week 0 were not observed (Figure 1).

\section{Fasting blood glucose}

Significant differences in blood glucose were seen at week 12 
between the control and the two sucrose-fed groups. No significant difference in mean fasting blood glucose was seen between the sucrosefed group and the sucrose $+\mathrm{AO}$ group (Figure 2). AO supplementation did not improve fasting blood glucose levels at the termination of the experimental timeline (Figure 2).

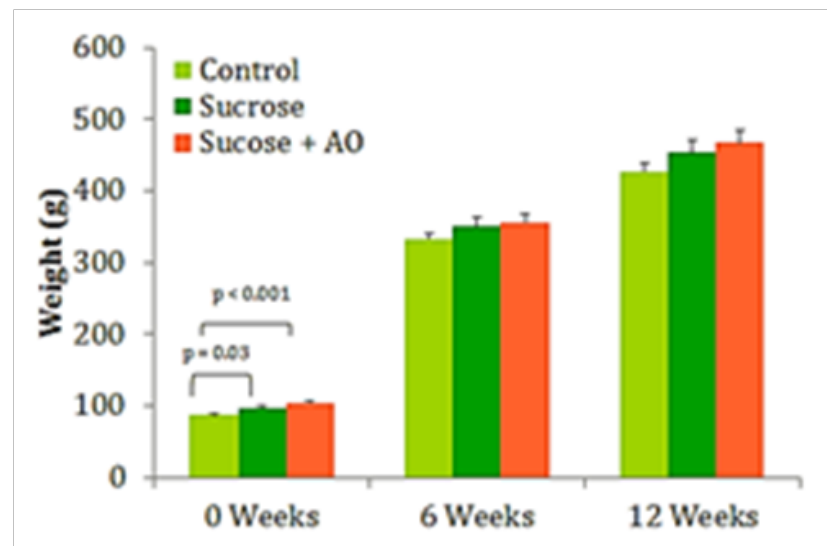

Figure I Mean body weight, effect of antioxidant supplementation on weight gain over I2weeks. Data are represented as means \pm standard deviations.

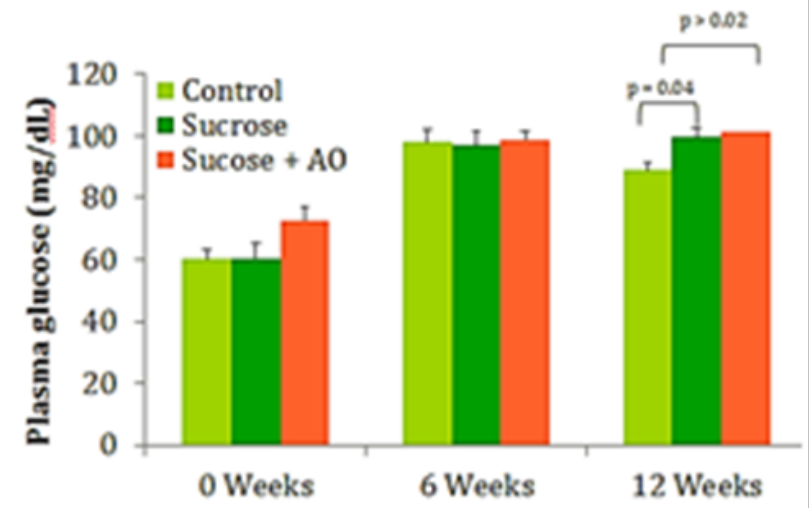

Figure 2 Fasting blood glucose, effect of antioxidant supplementation on fasting blood glucose levels over I2weeks. Data are represented as means \pm standard deviations.

\section{GFR}

Urine and plasma collected at weeks 0,6 , and 12 were used to calculate the creatinine clearance $(\mathrm{CC})$ at those intervals. No significant differences in $\mathrm{CC}$ were observed between the three groups at any time (Figure 3).

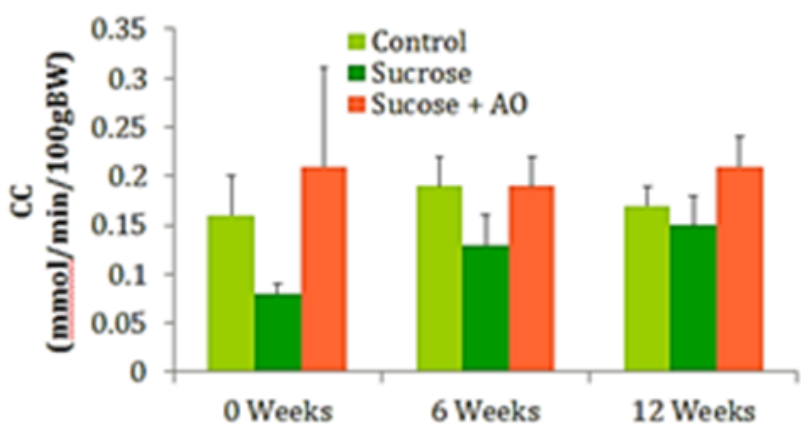

Figure 3 GFR; effect of antioxidant supplementation on GFR over 12weeks. Data are represented as means \pm standard deviations.

\section{Mean urine protein concentration}

The urine collected at weeks 0,6 , and 12 was used to calculate the protein concentration of the urine at those intervals. No significant differences in protein concentration were observed between the three groups at any time point (Figure 4).

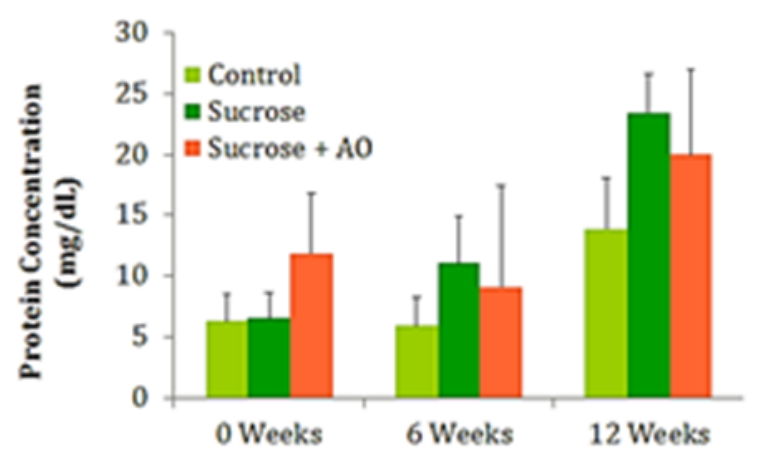

Figure 4 Protein concentration; effect of antioxidant supplementation on protein concentration over 12 weeks. Data are represented as means \pm standard deviations.

\section{eNOS and nNOS protein levels}

In the samples of the renal cortex of the control group, bands of 74 and $130 \mathrm{kDa}$ (monomers) and $320 \mathrm{kDa}$ (dimer) of eNOS were detected; medullary samples from the same group exhibited the same two monomers, but no dimer. Groups 2 and 3 fed the HS diet reveal significantly less of the $130-\mathrm{kDa}$ monomer in the cortex and less of the $74-$ and $130 \mathrm{kDa}$ monomers in the medulla as compared to the controls. Both cortical and medullary tissue from the experimental group exhibited significantly less of the 74-kDa monomer and total amount of eNOS content than both the control and the sucrose-fed group. No significant differences in total eNOS levels were noticed between the control and the sucrose only-fed group (Table 2). nNOS was expressed as 73 and $136 \mathrm{kDa}$ splice forms in both cortex and medulla. Cortical and medullary tissue from the experimental group exhibited significantly less nNOS content than renal tissues of the control group. Also, medullary tissue from the sucrose-fed groups had significantly lower nNOS content than controls. Significant differences between the sucrose and sucrose + AO group were not observed (Table 3 ).

Table 2 eNOS; Effect of antioxidant supplementation on eNOS/ $\beta$-actin ratio in the kidney cortex and medulla

\begin{tabular}{llll}
\hline & Control & Sucrose & Sucrose+AO \\
\hline Cortex & & & \\
$74 \mathrm{kDa}$ & $0.895 \pm 0.153$ & $1.218 \pm 0.176$ & $0.170 \pm 0.070^{*} \#$ \\
$130 \mathrm{kDa}$ & $1.059 \pm 0.072$ & $0.314 \pm 0.115^{*}$ & $0.126 \pm 0.026^{*}$ \\
$320 \mathrm{kDa}$ & $0.056 \pm 0.034$ & \pm & \pm \\
Total & $2.011 \pm 0.206$ & $1.532 \pm 0.255$ & $0.297 \pm 0.093^{*} \#$ \\
Medulla & & & \\
$74 \mathrm{kDa}$ & $0.991 \pm 0.125$ & $1.407 \pm 0.110^{*}$ & $0.106 \pm 0.022^{*} \#$ \\
$130 \mathrm{kDa}$ & $0.801 \pm 0.097$ & $0.280 \pm 0.084^{*}$ & $0.096 \pm 0.002^{*}$ \\
Total & $1.793 \pm 0.220$ & $1.688 \pm 0.190$ & $0.203 \pm 0.196^{*} \#$
\end{tabular}

*, $\mathrm{p}<0.05$ compared with Control; \#: $\mathrm{p}<0.05$ compared with Sucrose 
Table $3 \mathrm{nNOS}$, Effect of antioxidant supplementation on $\mathrm{nNOS} / \beta$-actin ratio in the kidney cortex and medulla

\begin{tabular}{llll}
\hline & Control & Sucrose & Sucrose+AO \\
\hline Cortex & & & \\
$73 \mathrm{kDa}$ & $1.237 \pm 0.228$ & $0.847 \pm 0.048$ & $0.540 \pm 0.058^{*}$ \\
$136 \mathrm{kDa}$ & $0.823 \pm 0.215$ & $0.729 \pm 0.045$ & $0.473 \pm 0.043^{*}$ \\
Total & $2.060 \pm 0.436$ & $1.576 \pm 0.084$ & $1.014 \pm 0.10 \mathrm{I}^{*}$ \\
Medulla & & & \\
$73 \mathrm{kDa}$ & $1.321 \pm 0.129$ & $0.729 \pm 0.092^{*}$ & $0.454 \pm 0.026^{*}$ \\
$136 \mathrm{kDa}$ & $0.794 \pm 0.143$ & $0.424 \pm 0.020^{*}$ & $0.276 \pm 0.042^{*}$ \\
Total & $2.115 \pm 0.268$ & $1.153 \pm 0.11 \mathrm{I}^{*}$ & $0.73 \mathrm{I} \pm 0.060^{*}$ \\
\hline
\end{tabular}

*, $\mathrm{p}<0.05$ compared with cortex

\section{Discussion}

The antioxidant (AO) diet given to type 2 diabetic (T2DM) rats prevented and/or delayed the progression of diabetic nephropathy, even with poor glycemic control. These results reveal evidence of molecular damage to the endothelium and specialized structures of the kidney that precedes the onset of diabetes, and early AO supplementation appears to safeguard the kidneys from some of the sheer forces that induce hyperfiltration in early-stage diabetic nephropathy. This suggests that early supplementation of AO's to the diet would be an inexpensive way to preserve renal function and prevent diabetic complications, even in the absence of good glycemic control.

Typically, weight gain and obesity precede the increase in fasting blood glucose and the subsequent development of T2DM. The $65 \%$ sucrose diet is able to induce significant increases in fasting blood glucose (FBG) by 12 weeks, yet they are below parameters for diabetes. The experimental AO diet was unable to significantly reduce FBG. Creatinine clearance is a marker for kidney function and possible damage. Early kidney damage may manifest with an increased creatinine clearance and subsequent decreases in creatinine clearance once the glomeruli and capillaries of the kidneys have been irreparably damaged. Proteinuria is another measure of kidney function and potential kidney damage. Abundant amounts of protein are dumped into the urine in individuals with diabetic nephropathy. These conventional measures of kidney function did not reveal significant differences in renal function. However, the NOS studies reveal significant differences in oxidative damage at the level of the endothelium. In order to understand the significance of the NOS study results, one must understand the two phases of renal functional decline in the progression of diabetic nephropathy. The early stage is characterized by renal microvascular vasodilation and thus hyperfiltration which begins to destroy the integrity of the glomerular membranes. In the later stages of the disease, renal function declines, structural damage appears, and the disease is unlikely to be reversible.

In mammals, the endothelial isoform of nitric oxide synthase (eNOS) is involved in signal generation and in the generalized control of vascular tone in the kidney. Increased expression of glomerular and cortical eNOS correlates with more severe vascular complications and degree of proteinuria. ${ }^{15}$ Therefore, the findings can be interpreted to mean that the AO diet led to the decrease in eNOS in the early phase of the onset of diabetic nephropathy, compared to the other 2 groups. This is beneficial because in the early phase of diabetic nephropathy, there is pre- and post-glomerular vasodilation that leads to increased renal blood flow and hyperfiltration. Consequently, lower levels of the vasodilator eNOS are protective in the early stages, given that less stress is exerted on the glomerular capillary membranes, ultimately attenuating the damage to the kidneys. Therefore, the $\mathrm{AO}$ diet appears to induce a protective decrease in the level of $\mathrm{NO}$, even before the onset of a disruption in the eNOS system by damage incurred from the hyperglycemic diet.

The neuronal isoform (nNOS) has been isolated in specialized cells of the kidney, such as the macula densa cells and the juxtaglomerular apparatus. In our model, cortical and medullary tissue from the experimental group exhibited significantly less nNOS content than renal tissues of the control group. Also, medullary tissue from the sucrose-fed groups had significantly lower nNOS than controls. Yamaleyeva et al. ${ }^{16}$ demonstrated that chronic treatment with the nNOS inhibitor L-VNIO conveys renal protection-that is, less inflammation throughout the kidneys and reduced decline in GFR-independent of changes in blood pressure in the salt-sensitive female strain described in their study. The findings of this study reflect our findings where nNOS levels were lowest in the diabetic rats administered the AO diet in the later stage of the progression of diabetic nephropathy. Again, the second phase of the progression of diabetic nephropathy is characterized by lower GFRs and irreversible kidney damage. We feel that if the study would have gone on longer, the creatinine clearance would have been found to be higher in our treated diabetic animals with corresponding depletion in the levels of nNOS.

Together, these findings imply that an AO diet has beneficial effects in preventing diabetic complications such as diabetic nephropathy in T2DM subjects when implemented at the earliest time of diagnosis. In future studies, it would be beneficial to consider other components of the NO pathway to solidify the claims made in this study. For example, observing the $\mathrm{AO}$ diet in vivo effects to investigate further into the renal microvascular vasodilatory effects to NO-mediated vasodilators. Future studies may observe fasting blood glucose, body weight gain, and more for a longer experimental period to prove claims made in this study. An antioxidant diet may be an inexpensive way to preserve renal function and delay the progression of diabetic complications, even without good glycemic control.

\section{Conclusion}

An antioxidant diet may be an inexpensive treatment for type 2 diabetics. In this study, exchanging a regular diet for a diet rich in antioxidants in the early stages of type 2diabetes was beneficial by preventing or delaying the progression of diabetic nephropathy, a common complication in diabetes, presumably by an initial decrease of endothelial nitric oxide synthase and a later decrease of neuronal nitric oxide synthase. Additional studies are needed to prove these findings and extend the claims made.

\section{Acknowledgements}

We thank the CORE for their support of this proposal with their funding of the CORE grant awarded to Lubka Ilieva for this project. We thank Aaron Wood, Nick Spittle, Sarah Decker, and Dr. Frank Schwartz M.D., F.A.C.E. for their contribution to the realization of this project.

\section{Conflict of interest}

The authors declare that there are no financial interests or conflicts of interest regarding the publication of this paper. 


\section{References}

1. Centers for Disease Control and Prevention. National diabetes statistics report: estimates of diabetes and its burden in the united states. US Department of Health and Human Services, Atlanta, Georgia; 2014:1-8.

2. Phillip M Hall. Prevention of progression in diabetic nephropathy. Diabetes Spectrum. 2006;19(1):18-24.

3. Kawazu S, Tomono S, Shimizu M, et al. The relationship between early diabetic nephropathy and control of plasma glucose in noninsulin-dependent diabetes mellitus. The effect of glycemic control on the development and progression of diabetic nephropathy in an 8-year follow-up study. J Diabetes Complications. 1994;8(1):13-17.

4. Singh DK, Winocour P, Farrington K. Oxidative stress in early diabetic nephropathy: fueling the fire. Nat Rev Endocrinol. 2010;7(3):176-184.

5. Slyvka Y, Wang Z, Yee J, et al. Antioxidant diet, gender and age affect renal expression of nitric oxide synthases in obese diabetic rats. Nitric Oxide. 2011;24(1):50-60.

6. Blouet C, Mariotti F, Mathe V, et al. Nitric oxide bioavailability and not production is first altered during the onset of insulin resistance in sucrose-fed rats. Exp Biol Med (Maywood). 2007;232(11):1458-1464.

7. Coimbra TM, Janssen U, Gröne HJ, et al. Early events leading to renal injury in obese Zucker (fatty) rats with type II diabetes. Kidney Int. 2000;57(1):167-182.

8. Prabhakar SS. Role of nitric oxide in diabetic nephropathy. Semin Nephrol. 2004;24(4):333-344.
9. Slyvka Y, Inman SR, Malgor R, et al. Protective effects of antioxidantfortified diet on renal function and metabolic profile in obese Zucker rat. Endocrine. 2009;35(1):89-100.

10. Opara EC. Role of oxidative stress in the etiology of type 2 diabetes and the effect of antioxidant supplementation on glycemic control. J Investig Med. 2004;52(1):19-23.

11. Vega López S, Devaraj S, Jialal I. Oxidative stress and antioxidant supplementation in the management of diabetic cardiovascular disease. J Investig Med. 2004;52(1):24-32.

12. Mekinová D, Chorváthová V, Volkovová K, et al. Effect of intake of exogenous vitamins $\mathrm{C}, \mathrm{E}$ and $\beta$-carotene on the antioxidative status in kidneys of rats with streptozotocin-induced diabetes. Nahrung. 1995;39(4):257-261.

13. Koo JR, Vaziri ND. Effects of diabetes, insulin and antioxidants on no synthase abundance and NO interaction with reactive oxygen species. Kidney Int. 2003;63(1):195-201.

14. Alfredo Rigalli, Veronica Di Loreto. Experimental surgical models in the laboratory rat. USA: CRC Press; 2009. 232p.

15. Hohenstein B, Hugo CP, Hausknecht B, et al. Analysis of NO-synthase expression and clinical risk factors in human diabetic nephropathy. Nephrol Dial Transplant. 2008;23(4):1346-1354.

16. Yamaleyeva LM, Lindsey SH, Varagic J, et al. Amelioration of renal injury and oxidative stress by the nNOS inhibitor L-VNIO in the salt-sensitive mRen2. Lewis congenic rat. J Cardiovasc Pharmacol. 2012;59(6):529-538. 